\author{
Ciaran Bolger \\ C. Carozzo \\ T. Roger \\ Linda McEvoy \\ Jabir Nagaria \\ Gerard Vanacker \\ Maurice Bourlion
}

\section{A preliminary study of reliability of impedance measurement to detect iatrogenic initial pedicle perforation (in the porcine model)}

Received: 13 January 2005

Revised: 27 June 2005

Accepted: 12 August 2005

Published online: 13 October 2005

(C) Springer-Verlag 2005
C. Carozzo $\cdot$ T. Roger

NeurosEcole Nationale Vétérinaire

de Lyonurgical Research and Development

Unit, Beaumont 69280 Marcy

l'EtoileHospital, 5201 Lyon, France

C. Bolger $\cdot$ L. McEvoy $(\bowtie) \cdot J$. Nagaria Neurosurgical Research and Development Unit, National Department for

Neurosurgery, Beaumont Hospital,

Dublin, Ireland

E-mail: 1mcevoy1@eircom.net

Tel.: + 353-1-8093929

Fax: + 353-1-8092302

G. Vanacker $\cdot$ M. Bourlion Spine Vision, Paris, France
Abstract Accidental perforation of the vertebral pedicle wall is a wellknown complication associated with standard approach of pedicle screw insertion. Depending on detection criteria, more than $20 \%$ of screws are reported misplaced. Serious clinical consequences, from dysesthesia to paraplegia, although not common, may result from these misplaced screws. Many techniques have been described to address this issue such as somatosensory evoked potentials, electromyography, surgical navigation, etc. Each of these techniques presents advantages and drawbacks, none is simple and ergonomic. A new drilling tool was evaluated which allows for instant detection of pedicle perforation by emission of variable beeps. This new device is based on two original principles: the device is integrated in the drilling or screwing tool, the technology allows real-time detection of perforation through two independent parameters, impedance variation and evoked muscular contractions. A preliminary animal study was conducted to assess the safety and efficacy of this system based upon electrical conductivity. A total of 168 manual pedicle drillings followed by insertion of implants were performed in 11 young porcine lumbar and thoracic spines.
The presence or absence of perforation detection, which defines the reliability of the device, was correlated with necropsic examination of the spines. Using this protocol the device demonstrated $100 \%$ positive predictive value, $96 \%$ negative predictive value, $100 \%$ specificity, and 97\% sensitivity. Of 168 drillings there were three $(1.79 \%)$ false-negatives, leading to a minor effraction, cranially in the intervertebral disks, nine $(5.36 \%)$ screw threads breaching the vertebral cortex when inserting screws, although preparation of the holes did not indicate any perforation, $34(36 \%)$ breaches detected by the instrument and not detected by the surgeon. These results confirm that the impedance variation detection capability of this device offers a simple and effective means to detect perforation in vertebral pedicle, prior to insertion of pedicle screws. Due to the porcine nerve root anatomy, it was not possible to evaluate the added benefit of cross-linking impedance and EMG detection. A future clinical study may further explore the subject of current study.

Keywords Porcine model $\cdot$ Pedicle screws - Radiographs - Pedicle breaches 


\section{Introduction}

Pedicle screw fixation has achieved significant popularity amongst spinal surgeons for both single- and multiplelevel spinal fusion [15]. Pedicle screw fixation plays an important role in the restoration of neural spaces in patients with a burst fracture [13], although it is more commonly used for treatment of degenerative spine, traumatic and non-traumatic abnormalities of the spine as well as spinal fixation after spinal tumors $[12,13]$.

There have been several studies published looking at both the placement and the pedicle cortical violation during screw placement, concluding that between 15 and $55 \%$ of pedicle screws breach the pedicle, causing a variety of complications such as, but not limited to, dysesthesia, paraparesis, or paraplegia $[3-5,9,10,15$, $18,20]$.

There have been many advances in the techniques employed for pedicle localization during pedicle screw placement and use of these techniques is dependent on the surgeon's preference during surgery. While each of these techniques aid in the navigation of the pedicles they also have their limitations. Somatosensory evoked potential (SSEP) has the drawback of being unable to assess the nerve roots during the insertion of the screws [7]. EMG studies on the other hand, while able to localize the nerve roots, requires a neurologist in the theatre during the pedicle preparation and is a rather invasive method [19]. The frequent use of intra-operative $\mathrm{X}$-rays can expose both the operative team and the patient to an excess of X-ray radiation. Other techniques include computer-aided frameless stereotaxis (surgical navigation) but this is an expensive tool, requiring preoperative CT scanning, registration technique, time consuming, and requires a wide range of experience and formal training [6]. Finally, the use of computed tomography for the assessment of pedicle screw position after surgery is too late [17].

Taking into the consideration the advantages and limitations of these aforementioned techniques, a new device has been designed to aid the surgeon during pedicle drilling. This new device is based on two original principles. First, that the tool should be as close to the normal surgical tools as possible and second, that the drilling tool would allow real-time detection of pedicle breaches. This is achieved by integrating the device into a standard pedicle perforation tool (Fig. 1) and by giving feedback to the surgeon every $0.5 \mathrm{~s}$ both through impedance variation and evoked muscle contractions. The device is, therefore, able to detect iatrogenic breach in the vertebral pedicle wall. It is a wireless perforation instrument, potentially improving safety of pedicle screw
Fig. 1 a Picture of the device dissembled. The disposable circuit board (a) is placed into the hollow handle (b) of the device. The tip of the device (c) is a standard drilling tool for pedicle preparation with the electromagnetic field sensor at the tip (d). b Assembled device with the LED activated

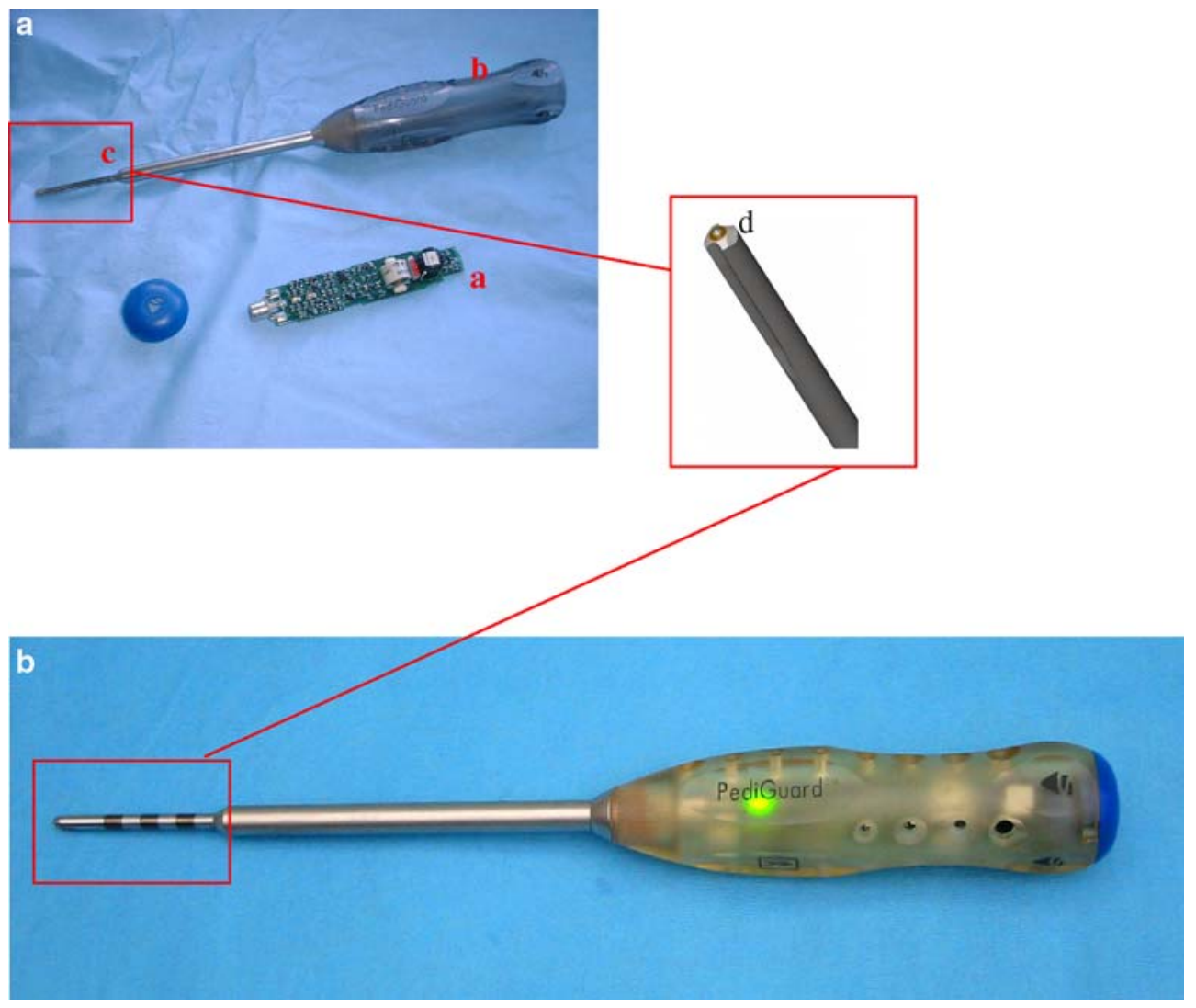


insertion without additional complexity for the surgeons. The device works on the principle of local tissue electrical impedance, which is monitored, at the tip of the instrument (Fig. 1). When the electrical conductivity at the distal part of instrument changes in the case of pedicle breech, it alerts the surgeon in the form of audio monitoring and visual feed back. The device is also able to stimulate muscle contraction that can be detected clinically or by EMG monitoring.

The aim of this study is to ascertain if impedance measurement can detect the presence of pedicle cortical breach and if this detection is superior to manual probing by the surgeon.

The device

The feature of this device includes bipolar electrodes that avoids any shunting effects and keep the measured electrical impedance independent of the insertion depth. In a same medium the electrical impedance remains constant while the instrument is advanced into the vertebral pedicle and variation occurs when the instrument passes through a boundary between two difference media.

The instrument itself consists of an awl with a hollow handle that accepts a built-in electronic printed circuit board (Fig. 1). The electronic components allow performing impedance measurement and translation to audible signal and colored light emitting diodes (LEDs) to be used as feedback every $0.5 \mathrm{~s}$ to the surgeon. In addition to this, the instrument also features a neurostimulator that provides a constant voltage and a limited current at a fixed frequency which can be used in conjunction with a standard EMG.

\section{Methods}

Using standard surgical techniques for pedicle preparation, 168 pedicle drillings were performed in a total of 11 pigs. The pigs were placed under general anesthetic as per customary veterinary protocol. Pedicle drilling was performed between T14 and L6 inclusive. Once the pedicle was prepared, pedicle screws were inserted.

All the drillings were performed by a qualified veterinarian, trained in the use of the perforation device. An independent assessor recorded the audible beeps and muscular contractions at the time of the drillings.

Breaches were identified, either intra-operatively by the veterinarian and the behavior of the instrument which was updated every $0.5 \mathrm{~s}$. Post-operatively the pigs were slaughtered and the individual vertebrae were inspected at autopsy for any breaches caused either by the placement of the pedicle screw or by the actual pedicle preparation, by two independent veterinarians.

The following parameters were computed for analysis purposes:

- False-positive: if the device tells that there is a breach through a rise in the auditory alert and after anatomic dissection there is none

- False-negative: if the device tells that there is a no breach, no rise in the auditory alert, and after anatomic dissection there is one

- Positive predictive value: probability of a breach if detection occurred

- Negative predictive value: probability of no breach if no detection occurred

- Specificity: probability of no-detection if there is no breach

- Sensitivity: probability of detection if there is a breach.
Fig. 2 Breaches detected on autopsy in pig vertebrae (a) lateral breach (b)anterior breach

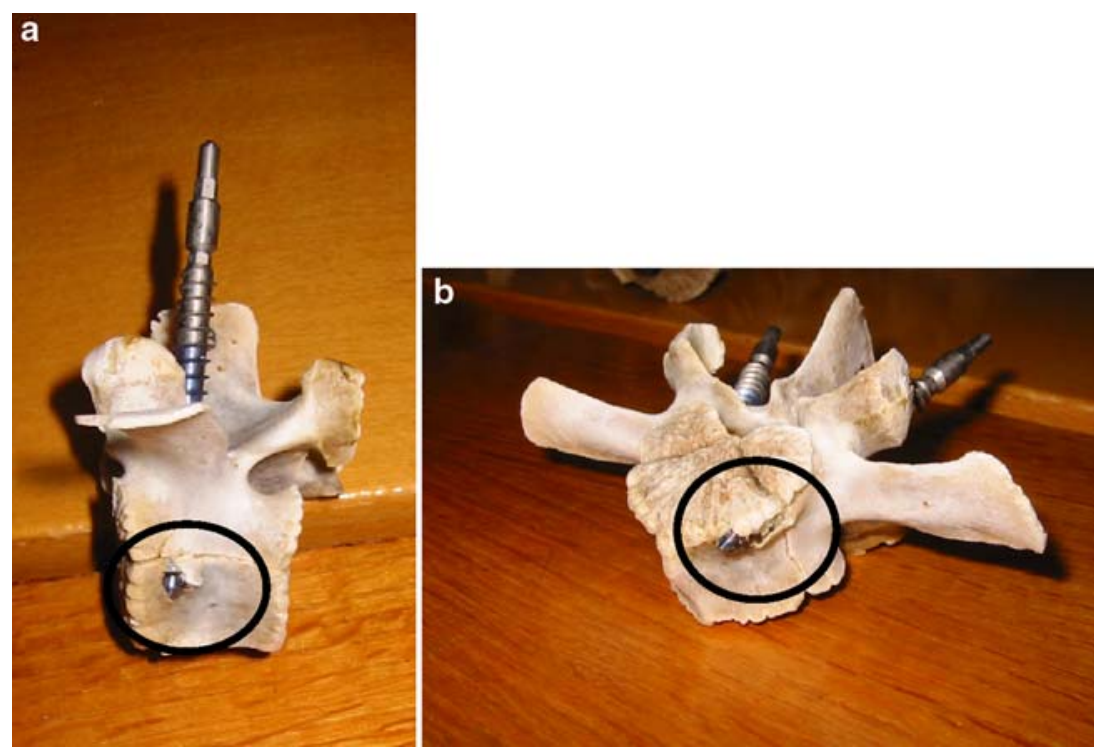


Statistical analysis was carried out using a standard statistical package (JMP, SAS Institute, USA), coupling the frequency of breaches, either detected by the device or the veterinarian, using visual inspection at autopsy as the gold standard. Using the Pearson's non-parametric test, we evaluated the correlation between the detection of breaches by the device and the veterinarian using pedicle probing. We also evaluated the device compared to anatomical dissection, for its ability to detect breaches.

\section{Results}

Of the 168 drillings there were a total of 93 breaches detected on anatomo-pathology investigations (Fig. 2). The Device detected $96 \%$ (90) of these pedicle breaches during the actual pedicle drilling, compared with $60 \%$ (56) detected by the veterinarian by pedicle probing. There were no pedicle breaches that were detected by the veterinarian and not by the Device (Pearson 72.8; $P<0.001)$ Table 1 .

There were three undetected breaches by the device, one breach medial $<2 \mathrm{~mm}$, one at the disc limit, and the third at the cranial disc. There were no breaches indicated by the device not confirmed on anatomical dissection. Negative predictive value $96 \%$, positive predictive value $100 \%$ (Pearson $156.3 ; \quad P<0.001$ ). Combining these results yields sensitivity for the device of $97 \%$ with a specificity of $100 \%$. The two breaches which lead to minor effraction in the inter-vertebral discs, which went undetected by the auditory alert of the device, were, however, detected on visual muscle contraction, another monitoring function of the device. However, since we are evaluating the impedance measurement aspect of the device alone these were recorded as "not detected" by the device Table 2 .

Table 1 Table of results of breaches detected by either the device or veterinarian during pedicle preparation

\begin{tabular}{ll}
\hline \#Drillings & 168 \\
\hline \#Breaches & $93(100 \%)$ \\
Detected by veterinarian & $56(60 \%)$ \\
Detected by device & $90(96.8 \%)$ \\
Detected by anatomic dissection & $93(100 \%)$ \\
\hline
\end{tabular}

Table 2 Statistical analysis of the device

\begin{tabular}{ll}
\hline Positive predictive value $^{\mathrm{a}}$ & $100 \%(90 / 90)$ \\
\hline Negative predictive value $^{\mathrm{b}}$ & $96 \%(168-93 / 168-90)$ \\
Specificity $^{\mathrm{c}}$ & $100 \%(168-93 / 168-93)$ \\
Sensitivity $^{\mathrm{d}}$ & $97 \%(90 / 93)$ \\
\hline
\end{tabular}

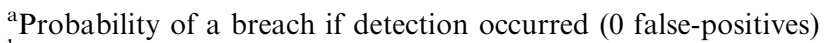

${ }^{\mathrm{b}}$ Probability of no breach if no detection occurred ( 3 false-negative)

${ }^{\mathrm{c}}$ Probability of no-detection if there is no breach

${ }^{\mathrm{d}}$ Probability of detection if there is a breach
As with pedicle probing by a surgeon, the device is designed as a pedicle preparation tool. Therefore, breaches that occur during the screw placement after pedicle drilling cannot be reflected. In this study, there were nine breaches caused by the threads of the screw alone that were identified on anatomical dissection. Neither the device nor pedicle probing detected these pedicles as breached prior to the screw placement.

\section{Discussion}

With advances in posterior spinal fixation instrumentation, increasing experience of pedicle anatomy among the spinal surgeons, and currently existing techniques for the accurate placement of pedicle screws, major changes have been seen in the management of spinal disorders. Posterior pedicle screw fixation system has gained popularity in the treatment of sclerotic and kypho-scoliotic deformities, tumors, trauma and spondylolisthesis, providing stability, correction of deformity and its maintenance, and increasing fusion rate $[8,12,13]$. This system is also in use in cases of lumbar degenerative disc disease without instability, to stop motion between the segments and increasing their fusion rates.

Several published series reported detailed anatomy of the cervical, thoracic, thoraco-lumbar, and lumbar pedicles and their relationship to the nearby vascular and neural structures $[2,11,14,16]$. Roy-Camille et al. [16] originally described the technique of pedicle screw placement in the thoracic spine. According to his technique, the entry point for screw placement was situated where two lines were intersecting each other, one passing vertically through the middle of the inferior facet and the other passing transversely through the middle of the transverse process. Vaccaro et al. [18] reported $41 \%$ and Rongming et al. [15] reported $55 \%$ pedicle cortex violation with Roy-Camille technique. In our study there were a total of $93(55 \%)$ breaches out of 168 drillings which is similar as recorded by previous studies. However, $96 \%$ (90) of these breaches were detected by the device, prior to screw insertion, thereby reducing the possibility of potential neurological complications, such as nerve root irritation, nerve root injury, and trauma to spinal cord. These complications are well documented in the literature, ranging in frequency from 1.1 to $10 \%$ $[3-5,9,15,16,18,20]$.

Acikbas et al. [1] in a retrospective review investigated the consequences of misplaced thoraco-lumbar pedicle screws on late spinal stability. In patients with screw misplacement, the findings were; inadequate correction of deformities, a progression of late kyphotic deformity, the evidence of significant motion on neutral-flexion and flexion-extension long-term radiographs and persistent moderate to severe back pain. Whereas in patients with properly placed screws, 
symptoms of late kyphotic deformities were not observed and the motion was evident only on neutralflexion radiographs. They concluded that correct placement of pedicle screws is required to maintain long-term spinal stability.

The main current existing techniques of pedicle localization and accurate placement of pedicle screws are; surgeon's knowledge (mechanical probing), surgical navigation, fluoro navigation, EMG and spinal cord monitoring, CT scan, fluoroscopy or plain X-ray films, patient's feed back and SSEP [6, 7, 17, 19]. The abovementioned techniques have their advantages and limitations. Some are operator dependent, others are expensive, may need a neurophysiologist in the operating room, and some are not easy to interpret. The operating team can be exposed to an excess of ionizing radiation due to multiple use of X-rays. Some of these procedures are not performed until post-operatively where it is possibly too late to know if there are misplaced pedicle screws.

Against this background, we have evaluated a new device, which in theory could reduce the number of pedicle breaches during pedicle screw insertion, without additional complexity for the surgeons, by alerting the surgeon to the possibility of a breach occurring. This device is based on two original principles; the device is integrated in the drilling or screening tool, and the technology allows real-time detection of perforation through two independent parameters, impedance variation and evoked muscular contractions. The device is therefore able to detect iatrogenic breach in the vertebral pedicle wall prior to screw insertion and therefore allows the surgeon to redirect. It is a wireless perforation instrument, working on the principle of local tissue electrical impedance, which is monitored, at the tip of the instrument. When the electrical conductivity at the distal part of the instrument changes in case of pedicle breech, it alerts the surgeon in the form of audio monitoring and visual feed back. The device is also able to stimulate muscles contraction that can be detected clinically or by EMG monitoring.

Due to the limitation with the anatomy of the porcine vertebral bodies and the location of the nerve roots, further studies are needed to accurately evaluate the benefit of the cross-linking impedance and the EMG detection of muscular contractions.

\section{References}

1. Acikbas SC, Arslan FY, Tuncer MR (2003) The effect of transpedicular screw misplacement on late spinal stability. Acta Neurochir 145:949-954

2. Berry JL, Moran JM, Berg WS, Steffee AD (1987) A morphometric study of human lumbar and selected thoracic vertebrae. Spine 12:362-367

3. Davne SH, Myers DL (1992) Complications of lumbar spine fusion with transpedicular instrumentation. Spine 17:184-189

4. Esses SI, Sachs BL, Dreyzin V (1993) Complication associated with technique of pedicle screw fixation. Spine 18:22312239

5. Gertzbein SD, Robbins SE (1990) Accuracy of pedicular screw placement in vivo. Spine 15:11-14

6. Glossop ND, Hu RW, Randle JA (1996) Computer-aided pedicle screw placement using frameless stereotaxis. Spine 21:2026-2034

7. Gundanna M, Eskenazi M, Bendo J, Spivak J, Moskovich R (2003)

Somatosensory evoked potential monitoring of lumbar pedicle screw placement for in situ posterior spinal fusion. Spine J 5:370-376
8. Liljenqvist UR, Halm HF, Link TM (1997) Pedicle screw instrumentation of the thoracic spine in idiopathic scoliosis. Spine 22:2239-2245

9. Lonstein JE, Denis F, Perra JH (1999) Complications associated with pedicle screws. J Bone Joint Surg Am 81:15191528

10. Lonstein JE, et al (1999) Complications associated with pedicle screws. J Bone Joint Surg Am 81:1519-1528

11. Ludwing SC, Kramer DL, et al (2000) Placement of pedicle screws in the human cadaveric cervical spine. Comparative accuracy of three techniques. Spine 25:1655-1667

12. Montesano PX, McLain RF, Benson DR (1991) Spinal instrumentation in the management of vertebral column tumours. Semin Orthop 6:237-246

13. Panjabi MM, Oda T, Wang J (2000) The effects of pedicle adjustments on neural spaces in burst fracture surgery. Spine 25:1637-1643

14. Panjabi MM, Takata K, Goel V, et al (1991) Thoracic human vertebrae: quantitative three-dimensional anatomy. Spine 16:888-901

15. Rongming $\mathrm{Xu}$, Nabil A Ebrahim, Yianjia Ou, Richard A Yeasting (1998) Anatomic considerations of pedicle screw placement in the thoracic spine. Spine 23:1065-1068
16. Roy-Camille R, Saillant G, Mazel C (1986) Plating of thoracic, thoracolumbar, and lumbar injuries with pedicle screw plates. Orthop Clin North Am 17:147-159

17. Sapkas GS, Papadakis SA, Stathakopoulos DP, Papagelopoulos PJ, Badekas AC, Kaiser JH (1999) Evaluation of pedicle screw position in thoracic and lumbar spine fixation using plain radiographs and computed tomography. Spine 24:1926

18. Vaccaro A, Rizzolo SJ, Balderston RA, et al (1995) Placement of pedicle screws in the thoracic spine Part II An anatomical and radiographic assessment. J Bone Joint Surg Am 77:1200-1206

19. Welch WC, Rose RD, Balzer JR, Jacobs GB (1997) Evaluation with evoked and spontaneous electromyography during lumbar instrumentation: a prospective study. J Neurosurg 87:397-402

20. West JL, Ogilvie JW, Bradford DS (1991) Complications of the variable screw plate pedicle screw fixation. Spine 16:576-579 